\title{
Evidence of linear lattice expansion and covalency enhancement in rutile $\mathrm{TiO} 2$ nanocrystals
}

\author{
Guangshe Li \\ Juliana Boerio-Goates \\ Brian F. Woodfield \\ Liping Li
}

Follow this and additional works at: https://scholarsarchive.byu.edu/facpub

Part of the Biochemistry Commons, and the Chemistry Commons

\section{Original Publication Citation}

Li, Guangshe, Juliana B. Goates, Brian F. Woodfield, and Liping Li."Evidence of linear lattice expansion and covalency enhancement in rutile TiO[sub 2] nanocrystals." Applied Physics Letters 85 (24): 259-261.

\section{BYU ScholarsArchive Citation}

Li, Guangshe; Boerio-Goates, Juliana; Woodfield, Brian F.; and Li, Liping, "Evidence of linear lattice expansion and covalency enhancement in rutile TiO2 nanocrystals" (2004). Faculty Publications. 416. https://scholarsarchive.byu.edu/facpub/416 accepted for inclusion in Faculty Publications by an authorized administrator of BYU ScholarsArchive. For more information, please contact ellen_amatangelo@byu.edu. 


\title{
Evidence of linear lattice expansion and covalency enhancement in rutile $\mathrm{TiO}_{2}$ nanocrystals
}

\author{
Guangshe Li, Juliana Boerio-Goates, and Brian F. Woodfield ${ }^{\mathrm{a})}$ \\ Department of Chemistry and Biochemistry, Brigham Young University, Provo, Utah 84602 \\ Liping Li \\ Department of Physics, Brigham Young University, Provo, Utah 84602
}

(Received 20 April 2004; accepted 9 July 2004)

\begin{abstract}
Lattice variations and bonding characteristics in rutile $\mathrm{TiO}_{2}$ nanocrystals were examined by x-ray diffraction and $\mathrm{x}$-ray photoelectron spectroscopy. With a reduction in the physical dimensions, rutile $\mathrm{TiO}_{2}$ nanocrystals show a linear lattice expansion and an anomalous covalency enhancement in apparent contradiction to the ionicity increase in $\mathrm{BaTiO}_{3}$ and $\mathrm{CuO}$ nanocrystals as reported recently by S. Tsunekawa et al. [Phys. Rev. Lett. 2000, 85, 3440] and V. R. Palkar et al. [Phys. Rev. B 1996, $\mathbf{5 3}, 2167]$. A surface defect dipole model is proposed to explain these physical phenomena in terms of the strong interactions among the surface dipoles that produce an increased negative pressure. The covalency enhancement is interpreted according to the critical properties of the increased Ti-O bond lengths in the expanded lattice. (C) 2004 American Institute of Physics.
\end{abstract}

[DOI: $10.1063 / 1.1790596$ ]

Metal-oxide nanocrystals have potential for or demonstrated applications in many technologies including solar energy conversion, batteries, and ductile ceramics. ${ }^{1}$ These materials have properties that are highly dependent on the physical dimensions or grain size. Extensive theoretical and experimental studies ${ }^{2}$ have shown that the dimensional reduction into the nanoscale regime produces distinct properties from the bulk, which is thought to originate from phonon confinement, additional surface phonons, or tensile surface stresses. The reduction in grain size is also generally followed by certain variations in lattice parameters. From a solid-state physics viewpoint, the lattice dimensions directly determine the band structure and, consequently, the physical properties of the solids since the variations in lattice can lead to a significant shift of the Brillouin zone. Metal-insulator transitions observed in lower-dimensional solids are a good example, illustrating the importance of lattice modulations. ${ }^{3}$ However, the origin of size-induced changes in the lattice volume of metal-oxide nanocrystals is still not clearly understood.

In contrast with the lattice contraction in metal nanocrystals, ${ }^{4}$ most metal-oxide nanocrystals exhibit lattice expansion as the particle size is reduced. ${ }^{5,6}$ This is somewhat surprising since it is counter to general physical observations for small particles ${ }^{7}$ in which surface stresses cause small particles to compress. Tsunekawa et al. ${ }^{5}$ proposed that the lattice expansion in metal-oxide nanocrystals is due to the valence reduction such as from $\mathrm{Ce}^{4+}$ to the larger $\mathrm{Ce}^{3+}$ in $\mathrm{CeO}_{2}$ nanocrystals, but Palkar et al. ${ }^{6}$ found that there was no oxygen loss as the particle size was reduced. Furthermore, in certain transition-metal oxides, it has also been reported ${ }^{6}$ that there is an ionicity increase as the particle size is reduced, which is explained on the basis of a pressure-induced covalency increase. This conclusion is questionable, since in certain solids that are primarily ionic in nature, ionicity enhancement occurs at high pressures. ${ }^{8}$ Rutile $\mathrm{TiO}_{2}$ is a model

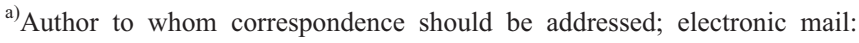
brian_woodfield@byu.edu compound that shows approximately $63 \%$ ionic nature in the $\mathrm{Ti}-\mathrm{O}$ bonding as deduced from the electronegativity values of $\mathrm{Ti}$ and $\mathrm{O}$, and has a close structural link to high-pressure phases of many metal oxides including $\mathrm{GeO}_{2},{ }^{9} \mathrm{ZrO}_{2}$, and $\mathrm{HfO}_{2}{ }^{10}$ as well as the low-pressure earth's mantle material, stishovite $\mathrm{SiO}_{2}{ }^{11}$ Of its various analogues, rutile $\mathrm{TiO}_{2}$ is among the few that can be stabilized at nanoscale sizes under ambient conditions with a typical size effect being demonstrated by a significant decrease in the transition pressure to a phase of $\mathrm{PbO}_{2}$-type $\mathrm{TiO}_{2}{ }^{12}$ This has also led to the possibility of finding more high-pressure polymorphs including $\alpha-\mathrm{PbO}_{2}$-type and baddeleyite-structured $\mathrm{TiO}_{2}$. A precise determination of the size dependence of the lattice volumes and bonding characteristics of rutile $\mathrm{TiO}_{2}$ nanocrystals is thus expected to improve our understanding on the nanophysics, size-related properties (e.g., quantum effects), and even certain geological processes.

In this letter, we report a linear lattice expansion as a function of particle size in rutile $\mathrm{TiO}_{2}$ nanocrystals that demonstrates critical quantum-size effects.

Highly pure rutile nanoparticles were prepared by a hydrothermal method. ${ }^{13}$ X-ray diffraction (XRD) data of the rutile nanoparticles were measured at room temperature at a scan rate of $0.2^{\circ} 2 \theta / \mathrm{min}$. The lattice parameters for the samples were calculated by least-squares methods. The average grain size $(D)$ was measured from the most intense XRD peak (110) using the Scherrer formula. ${ }^{13}$ The ionic characteristics were studied by determining the binding energies of the $\mathrm{O} 1 s$ and Ti $2 p$ electrons using X-ray photoelectron spectroscopy (XPS). The binding-energy data are calibrated with the $\mathrm{C} 1 s$ signal at $284.6 \mathrm{eV}$.

XRD and TEM have already confirmed the formation of single-phase rutile $\mathrm{TiO}_{2}$ nanocrystals in a rodlike shape. ${ }^{13}$ Figure 1 shows the main diffraction peak (110) on an expended scale, where the broadening effects are clearly seen. The strain and particle size are two predominant factors controlling the broadenings of the diffraction peaks. ${ }^{14}$ The effective strain is calculated using the Williams and Hall theorem $^{15}$ 


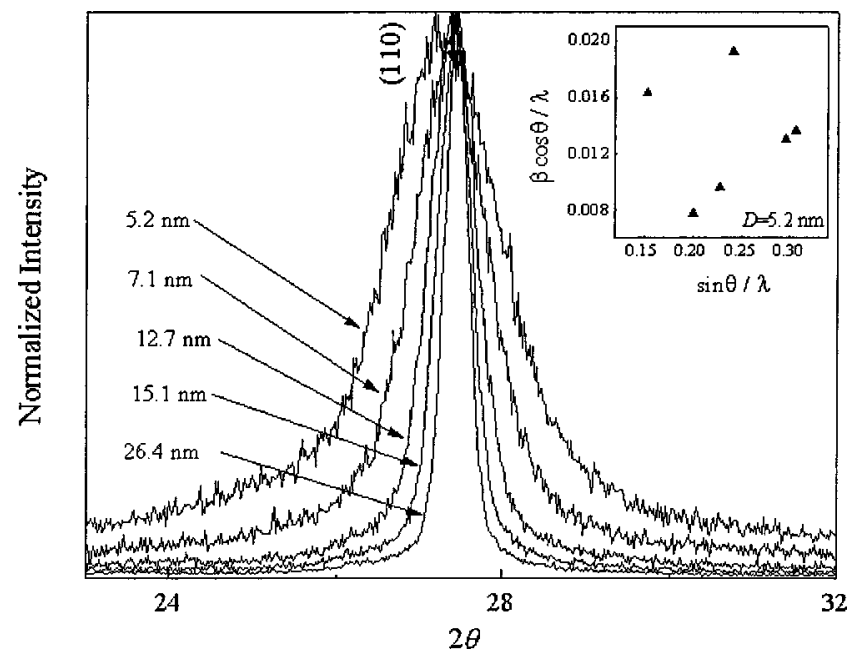

FIG. 1. Normalized diffraction peak (110) for rutile $\mathrm{TiO}_{2}$ nanocrystals at the indicated grain sizes. Inset is the relationship between $(\beta \cos \theta) / \lambda$ and $(\sin \theta) / \lambda$.

$$
\frac{\beta \cos \theta}{\lambda}=\frac{1}{D}+\frac{\eta \sin \theta}{\lambda},
$$

where $\beta$ is the full width at half maximum (FWHM), $\theta$ is the diffraction angle, $\lambda$ is the x-ray wavelength, $D$ is the effective particle size, and $\eta$ is the effective strain. A typical plot of $(\beta \cos \theta) / \lambda$ vs $(\sin \theta) / \lambda$ for six intense peaks of $5.2 \mathrm{~nm}$ rutile is shown in the inset of Fig. 1. The scattered data points strongly demonstrate the absence of any significant strain in our rutile $\mathrm{TiO}_{2}$ nanocrystals. Consequently, the average grain size of the rutile nanocrystals was calculated using only the peak broadenings, which will be described in more detail elsewhere. Also indicated in Fig. 1, the (110) peak shows a significant shift towards lower diffraction angles as the particle size is reduced. This is associated with the lattice expansion and has already been found in $\mathrm{CeO}_{2}$ nanocrystals containing dopants larger than $\mathrm{Ce}^{4+}$, such as $\mathrm{Nd}^{3+16}$

Structural refinements using a least-squares method indicate the lattice volume of rutile $\mathrm{TiO}_{2}$ nanocrystals increases monotonically with a reduction in grain size $(D)$ (Fig. 2).

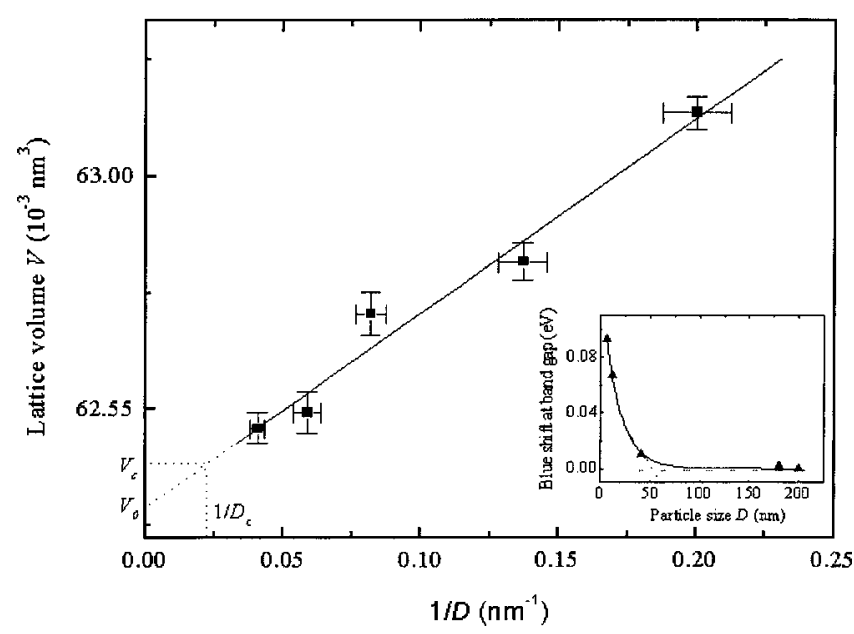

FIG. 2. Grain-size dependence of the lattice volume for rutile $\mathrm{TiO}_{2}$ nanocrystals (ם). Inset shows grain-size dependence of the blueshifts of the band gap compared with that of the bulk $\mathrm{TiO}_{2}$ crystallites (A) (see Ref. 22).

Downloaded 13 Feb 2009 to 128.187.0.164. Redistribution subjed
The lattice volume $(V)$ size dependence was fitted to the following linear equation:

$$
V=V_{0}+\frac{A}{D}
$$

where $V_{0}=6.236 \times 10^{-2} \mathrm{~nm}^{3}$ and $A=3.75(0) \times 10^{-3} \mathrm{~nm}^{4}$. The occurrence of a linear lattice expansion can be related to a negative interface pressure, but this is different from the positive surface pressure that is associated with the lattice contractions in metal nanocrystals with a predominant surface stress effect via $f_{r r}=\frac{1}{2} r \cdot p$ (where $f_{r r}$ is the surface stress and $p$ is the hydrostatic pressure due to the surface of radius $r){ }^{4}$ Tsunekawa et al. ${ }^{5}$ and Ayyub et al. ${ }^{17}$ have proposed several reasons for the lattice expansions appearing in certain metal-oxide nanocrystals according to (i) the valence reduction as in $\mathrm{CeO}_{2}$, ${ }^{5}$ and (ii) the unpaired electronic orbitals at the outer surface as in $\mathrm{Fe}_{2} \mathrm{O}_{3} \cdot{ }^{17}$ However our XPS and EPR results do not show any evidence of $\mathrm{Ti}^{3+}$ or oxygen vacancies in our rutile $\mathrm{TiO}_{2}$ nanocrystals. Here, we propose a surface defect dipole model to explain the negative pressure observed for the rutile nanocrystals.

Ideally, in the highly symmetric $\mathrm{Ti}-\mathrm{O}_{6}$ octahedra, the centers of positive and negative charges are located at the octahedral sites. However, rutile $\mathrm{TiO}_{2}$ has been shown to have intrinsic polarization fields in the lattice. ${ }^{18}$ That is, the coordinated oxygen ions occupy positions where crystal electrical fields may occur causing the ions to become polarized. ${ }^{19}$ The titanium atoms are distributed with some amount of disorder at several sites adjacent to the octahedral sites due to the low-energy barrier between these sites. ${ }^{20}$ Therefore, titanium atoms can move off the centers of the $\mathrm{Ti}-\mathrm{O}_{6}$ octahedra building blocks and form dipoles. Because of the smaller cell volume of the rutile lattice and two equivalent molecules for each cell, stronger interactions between dipoles of the $\mathrm{Ti}-\mathrm{O}_{6}$ octahedra can be expected, which most likely leads to a highly oriented array of dipoles with a directional character similar to those found in most titanate ferroelectrics and piezoelectrics. ${ }^{21}$

There are several factors that can considerably enhance the strength of the surface dipoles in rutile nanocrystals. With decreasing grain size, there will be a larger fraction of titanium atoms appearing on the surface with lower coordination compared with the six-fold coordinated $\mathrm{Ti}$ in the bulk phase. Consequently, surface titanium atoms will most likely show a significant increase in the number of dangling bonds, which could be compensated by the absorption of some reactive molecules, such as $\mathrm{H}_{2} \mathrm{O}$, by forming terminal hydration layers as was confirmed by our TGA-DSC and IR measurements (not shown), causing a significant distortion around the Ti ions. Consequently, the negative centers will show a significant downward shift towards the grain interior relative to the positive centers of the surface titanium atoms. This shift of the negative centers is expected to produce enhanced surface defect dipoles that are perpendicular to the outer surface of each rutile nanocrystal. The strong dipoledipole interactions would lead to a roughly parallel array of the dipoles yielding larger defect dipoles on the surface layer of each nanocrystal. The strong repulsive interactions of the parallel surface defect dipoles account for the negative pressure on the rutile nanocrystals. Even though the nanocrystals may also show some positive pressure due to surface stresses as has been indicated in metal nanocrystals, ${ }^{4}$ for the present to AIP license or copyright; see http://apl.aip.org/apl/copyright.jsp 


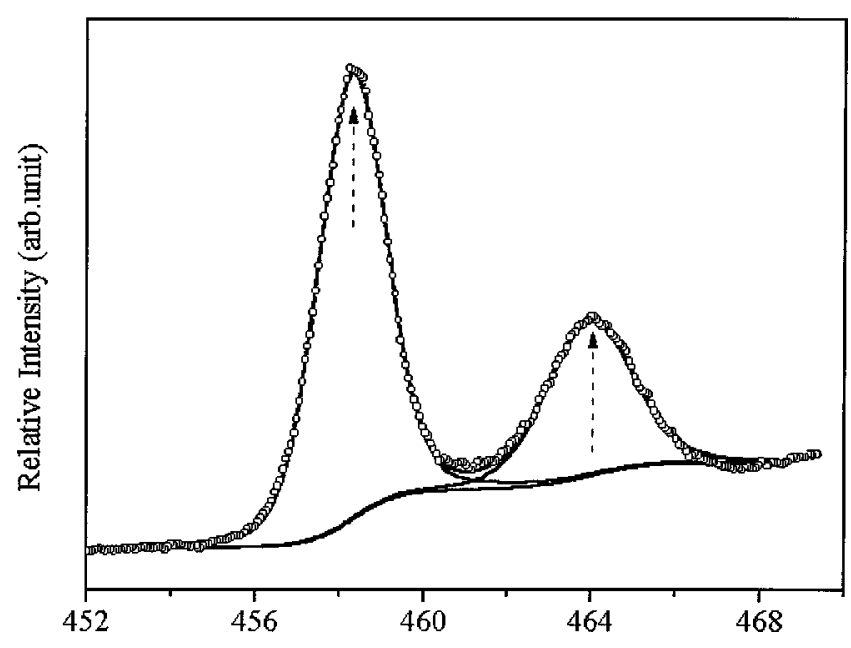

FIG. 3. XPS Ti $2 p$ core-level spectra for $7.9 \mathrm{~nm}$ rutile nanocrystals.

rutile nanocrystals, the negative pressure is evidently much larger as the particle size is reduced below some critical dimension. The net negative pressure is the primary reason for rutile nanocrystals having a larger equilibrium volume than that of the bulk phase. Similar defect dipoles have been proposed on the grain surfaces of $\mathrm{Ba}_{4} \mathrm{Ti}_{3} \mathrm{O}_{12}$ nanocrystals and $(\mathrm{Ba}, \mathrm{Sr}) \mathrm{TiO}_{3}$-based oxides. ${ }^{21}$

Now let us return to our linear equation for lattice expansion in rutile nanocrystals. Equation (2) can be rewritten as follows:

$$
V=V_{0}+\frac{\left(V_{c}-V_{0}\right) \cdot D_{c}}{D},
$$

where $V_{0}\left(=6.236 \times 10^{-2} \mathrm{~nm}^{3}\right)$ denotes an imaginary bulk lattice infinitely large and $D_{c}$ and $V_{c}$ are the critical particle size and lattice volume when the external forces from the surface dipoles and surface tension are balanced. As the particle size increases beyond $D_{c}$, the relative surface area and surface tension will become extremely small and the lattice volume at $D \geqslant D_{c}$ can be taken as a constant; that is, $V_{c}$ will have a value much closer to the actual lattice volume of 6.243 $\times 10^{-2} \mathrm{~nm}^{3}$ for the bulk phase of rutile $\mathrm{TiO}_{2}$. If we assume this value for $V_{c}$, Eq. (3) will yield a critical size of $D_{c}$ $=54 \mathrm{~nm}$. This critical size, obtained by our linear lattice expansion, is in excellent agreement with that of approximately $50 \mathrm{~nm}$ as determined by the photoluminescence method ${ }^{22}$ below which a significant blueshift is observed in the absorption spectra relative to those of bulk phases, indicating a quantum-size effect (see the inset of Fig. 2).

The lattice expansion in rutile nanocrystals is also associated with changes in the chemical bonding. The Ti $2 p$ spectra of $7.9 \mathrm{~nm}$ rutile $\mathrm{TiO}_{2}$ (Fig. 3) consist of well-defined Ti $2 p_{1 / 2}$ and Ti $2 p_{3 / 2}$ photoelectron signals that are located near 464 and $458 \mathrm{eV}$, respectively. No shoulders associated with $\mathrm{Ti}^{3+}$ or $\mathrm{Ti}^{2+}$ were observed at the lower energy sides. The spin-orbital splitting between these peaks is nearly constant at approximately $5.6 \mathrm{eV}$, which is comparable with the value of $5.74 \mathrm{eV}$ for nanoporous anatase $\mathrm{TiO}_{2}{ }^{23}$ Taking the $\mathrm{O} 1 \mathrm{~s}$ binding energy for the bulk oxygen species as a reference, the bonding energy difference $(\Delta E)$ between $\mathrm{O} 1 s$ and $\mathrm{Ti}$
$2 p_{3 / 2}$ was calculated by fitting the photoelectron peaks. It was found that with decreasing particle size from 17.8 to 5.4 $\mathrm{nm}, \Delta E$ decreased from $71.40(2)$ to $70.54(2) \mathrm{eV}$, which clearly indicates a decrease in the relative ionicity that has also been demonstrated by a nonempirical electronicstructure calculation. ${ }^{24}$ The covalency enhancement is closely related to the change in the electronic transfer ability during the lattice expansion. The covalent $\mathrm{Ti}-\mathrm{O}$ bond distance is $0.202 \mathrm{~nm}$, which is nearly the same as that for the ionic Ti-O bond length $(0.201 \mathrm{~nm}){ }^{5}$ The increased $\mathrm{Ti}-\mathrm{O}$ bond lengths during the lattice expansion might provide a higher barrier for retarding the electronic transfer between $\mathrm{Ti}$ and $\mathrm{O}$ by reducing the ability to donate or accept electrons between $\mathrm{Ti}$ and $\mathrm{O}$ atoms.

This work was supported with a grant (DE-FG0301ER15235) from the U.S. Department of Energy. The authors wish to thank Professor Alexandra Navrotsky, University of California at Davis, and Professor Frances Hellman, University of California at Berkeley, for many fruitful discussions as part of our on-going collaboration.

${ }^{1}$ B. O'Regan and M. Gratzel, Nature (London) 353, 737 (1991); W. Li, H. Osora, L. Otero, D. C. Duncan, and M. A. Fox, J. Phys. Chem. A 102, 5333 (1998); L. Kavan, K. Kratochvilova, and M. Gratzel, J. Electroanal. Chem. 394, 93 (1995).

${ }^{2}$ G. L. Freg, R. Tenne, M. J. Matthews, M. S. Dresselhaus, and G. Dresselhaus, Phys. Rev. B 60, 2883 (1999); S. F. Ren and G. Qin, Solid State Commun. 121, 171 (2002).

${ }^{3}$ D. Jerome and H. J. Schulz, Adv. Phys. 51, 293 (2002).

${ }^{4}$ A. Taneda and Y. Kawazoe, J. Magn. Soc. Jpn. 23, 679 (1999).

${ }^{5}$ S. Tsunekawa, K. Ishikawa, Z. Q. Li, Y. Kawazoe, and A. Kasuya, Phys. Rev. Lett. 85, 3440 (2000).

${ }^{6}$ V. R. Palkar, P. Ayyub, S. Chattopadhyay, and M. Multani, Phys. Rev. B 53, 2167 (1996).

${ }^{7}$ C. W. Mays, J. S. Vermaak, and D. Kuhlmann-Wilsdorf, Surf. Sci. 12, 134 (1968).

${ }^{8}$ J. R. Chelikowsky and J. K. Burdett, Phys. Rev. Lett. 56, 961 (1986).

${ }^{9}$ V. B. Prakapenka, L. S. Dubrovinsky, G. Shen, M. L. Rivers, S. R. Sutton, V. Dmitriev, H. P. Weber, and T. Le Bihan, Phys. Rev. B 67, 132101 (2003).

${ }^{10}$ N. A. Dubrovinskaia, L. S. Dubrovinsky, R. Ahuja, V. B. Prokopenko, V. Dmitriev, H. P. Weber, J. M. Osorio-Guillen, and B. Johansson, Phys. Rev. Lett. 87, 275501 (2001).

${ }^{11}$ D. Andrault, R. J. Angel, J. L. Mosenfelder, and T. Le Bihan, Am. Mineral. 88, 301 (2003).

${ }^{12}$ J. S. Olsen, L. Gerward, and J. Z. Jiang, J. Phys. Chem. Solids 60, 229 (1999).

${ }^{13} \mathrm{G}$. Li, L. Li, J. Boerio-Goates, and B. F. Woodfield, J. Mater. Res. 18, 2664 (2003).

${ }^{14}$ S. B. Qadri, J. P. Yang, E. F. Skelton, and B. R. Ratna, Appl. Phys. Lett. 70, 1020 (1997).

${ }^{15}$ G. K. Williamson and W. H. Hall, Acta Metall. 1, 32 (1953).

${ }^{16}$ L. Li, X. Lin, G. Li, and H. Inomata, J. Mater. Res. 16, 3207 (2001).

${ }^{17}$ P. Ayyub, V. R. Palkar, S. Chattopadhyay, and M. Multani, Phys. Rev. B 51, 6135 (1995).

${ }^{18}$ B. O’Regan and M. Gratzel, Nature (London) 353, 737 (1991).

${ }^{19}$ M. Birkholz, Z. Phys. B: Condens. Matter 96, 325 (1995).

${ }^{20}$ R. J. Betsch, H. L. Park, and W. B. White, Mater. Res. Bull. 26, 613 (1991).

${ }^{21}$ A. Q. Jiang, G. H. Li, and L. D. Zhang, J. Appl. Phys. 83, 4878 (1998).

${ }^{22}$ M. Anpo, T. Shima, S. Kodama, and Y. Kubokawa, J. Phys. C 91, 4305 (1987).

${ }^{23}$ S. Sodergren, H. Siegbahn, H. Rensmo, H. Lindstriom, A. Hagfeldt, and S. E. Lindquist, J. Phys. Chem. B 101, 3087 (1997).

${ }^{24}$ V. A. Vergazov, A. V. Leko, and R. A. Evarestov, Phys. Solid State 41, 1286 (1999). 